Segmental arterial mediolysis with mesangial cell hyperplasia A review with supplementary comments concerning its pathogenesis

Slavin, Richard E.; Leifsson, Páll Skúli

Published in:

Interventional Cardiology

DOI:

10.4172/Interventional-Cardiology.1000576

Publication date:

2017

Document version

Publisher's PDF, also known as Version of record

Document license:

Unspecified

Citation for published version (APA):

Slavin, R. E., \& Leifsson, P. S. (2017). Segmental arterial mediolysis with mesangial cell hyperplasia: A review with supplementary comments concerning its pathogenesis. Interventional Cardiology, 9(4), 181-190.

https://doi.org/10.4172/Interventional-Cardiology.1000576 


\section{Segmental arterial mediolysis with mesangial cell hyperplasia; A review with supplementary comments concerning its pathogenesis}

\begin{abstract}
Background: Segmental arterial mediolysis (SAM) a rare arteriopathy causing massive bleeding or ischemic symptoms, is suspected of representing a vascular disease of the peripheral sympathetic nervous system. It is initiated by the supra physiological release of norepinephrine from the efferent branches of the peripheral sympathetic nerves that innervate the large and medial sized muscular arteries targeted in SAM. Recognized stimuli for this response are iatrogenic sympathomimetic agonists and some B-2 agonists. However, these stimuli were not always apparent in published cases of SAM casting doubt on this hypothesis.
\end{abstract}

Methods and findings: SAM, reported in kidneys of slaughtered pigs, was believed to represent a dysfunctional development in a fight and flight response. Additionally some stenotic renal arteries described in cases of pheochromocytomas reportedly were caused by arterial spasm and fibromuscular dysplasia changes found in the evolution of SAM. Varying degrees of mesangial cell hyperplasia accompanied SAM in swine, dog and scattered human cases of SAM. Segmental sclerosis of glomerular loops accompanied the mesangial cell hyperplasia in a few cases of SAM. The hyperplasia was not extensive so that its role in regulating blood flow and urine volume through glomerular capillaries was probably inconsequential. It represented a collateral lesion created by SAM's norepinephrine driven pathogenesis.

Conclusion: These findings provide indirect evidence that SAM additionally may be initiated by conditions causing the adrenal medulla to release supra physiologic levels of circulating norepinephrine. Supra physiologic release of norepinephrine from the peripheral sympathetic nerves also can cause mesangial hyperplasia that can be accompanied with segmental glomerular loop sclerosis-making it another precursor lesion of focal segmental glomerulosclerosis.

Keywords: Norepinephrine, Renal artery stenosis, Fibromuscular dysplasia, Mesangial cell hyperplasia, Fight and flight response, Pheochromocytoma, Focal segmental glomerulosclerosis

\section{Introduction}

Segmental arterial mediolysis (SAM) is relatively rare non-systemic arteriopathy that occurs in large and medium sized muscular arteries located in the abdominal cavity, retroperitoneum, brain base and heart. It can cause catastrophic hemorrhages in its initial injurious phase through ruptured gap-aneurysms created from arterial zones of extensive trans mural mediolysis and intimal loss $[1,2]$. Ischemic symptoms may develop in its secondary reparative phase. These stem from the florid proliferating granulation tissue that mends the arterial lesions created in the injurious phase-the shearing lesion formed at the adventitial-medial junction and media muscle loss created by mediolysis or apoptosis. Reparative changes create
Richard E. Slavin ${ }^{1 *}$ and Pall S. Leifsson²

${ }^{1}$ Legacy Research Institute, Department of Pathology, Legacy Emanuel Hospital and Health Center, Portland, OR, USA ${ }^{2}$ Department of Veterinary and Animal Sciences, University of Copenhagen, Copenhagen, Denmark

*Author for correspondence: Tel: +15036380175

E-mail: reslavin@comcast.net Submitted: July 27, 2017

Accepted: August 08, 2017

Published online: August 14, 2017 
sequelae-dissecting hematomas, arterial stenosis and thrombosis, persistent aneurysms and if extensive fibromuscular dysplasia [2-6]. Significant hemorrhages can also occur in the reparative phase originating from dissecting hematomas but this event may be delayed for months to two or more years after the onset of SAM and have been provoked by the misplacement of stents into stenotic arteries [7]. Organ injury such as pancreatic hemorrhages and renal infarcts may also arise from involved arteries entering these organs [1,7]. SAM in its two phases can be subclinical discovered serendipitously on radiologic examinations, in surgical resections or at autopsy [5]. Reparative medial lesions may be asymptomatic and non-visible in angiographic studies [7]. SAM's varying clinical presentations are directly related to the type, intensity and site of the injurious lesions and their reparative granulation tissue within targeted arteries. The age range of this entity ranges from the fetus to the elderly. Abdominal hemorrhages are most often encounter in the elderly with an equal sexual distribution while ischemic lesions, created by arterial stenosis often presenting as fibromuscular dysplasia are encountered more often in younger adult female patients [6]. Dissecting hematomas in coronary arteries are the most common symptom-generating lesion of SAM in infants, children and young adults.

SAM has also been reported greyhounds and pigs $[8,9]$. Mesangial cell hyperplasia accompanied SAM in these animals. This lesion was also rarely identified in human SAM [1]. This article will explain how mesangial cell hyperplasia is related to SAM through a shared pathogenesis and how swine SAM led to the consideration of a supplementary pathogenic pathway for SAM caused by the supra physiologic production of circulating norepinephrine from the adrenal medulla.

\section{Methods and Materials}

The figures utilized in this article are derived from the multiple cases of SAM reported by the authors in references [1-10]. Their journal origins are listed in the figure descriptions. The figures chosen in this review article were selected to represent all of the evolutionary morphologic pathways that this disease could pursue in its injurious and reparative phases. They represent a parallel histologic view of SAM's clinical and angiographic presentations. Since SAM is a relatively unknown disorder the figures also serve as a lexicon for physicians, unfamiliar with this disease, charged with histologically diagnosing SAM-its morphologic identification being the gold standard for its diagnosis [6].

\section{Discussion}

In a recent article SAM was reported in the interlobar and arcuate arteries of kidneys in 36 pigs slaughtered in an abattoir in Jutland Denmark [9]. The kidneys grossly exhibited cortical infarct-like lesions that as a public health measure were histologically examined to rule out an infectious process (septic emboli from an infectious endocarditis). Half of these kidneys also showed the mesangial cell hyperplasia illustrated in Figure 1A. Its significance was uncertain. Was it normal, a consequence of other illnesses not revealed because the tissue diagnosis was limited to the kidney or were we witnessing another pathological manifestation of SAM? To test the latter supposition, the kidney of the greyhound dog sacrificed 4 days following ractopamine intoxication whose necropsy showed SAM involving the hepatic artery, renal hilar and lobar arteries and pericardial coronary arteries and their large penetrating branches was reexamined. Focal mesangial cell hyperplasia was also found in the kidney of this dog (Figure 1B). Mesangial cell hyperplasia was also reported in humans with SAM involving the kidneys (Figure 2) [1]. These combined findings favors the belief that mesangial cell hyperplasia does indeed represent another pathologic alteration developing in SAM. A proposed common pathogenesis for both entities, described below, supports this hypothesis.

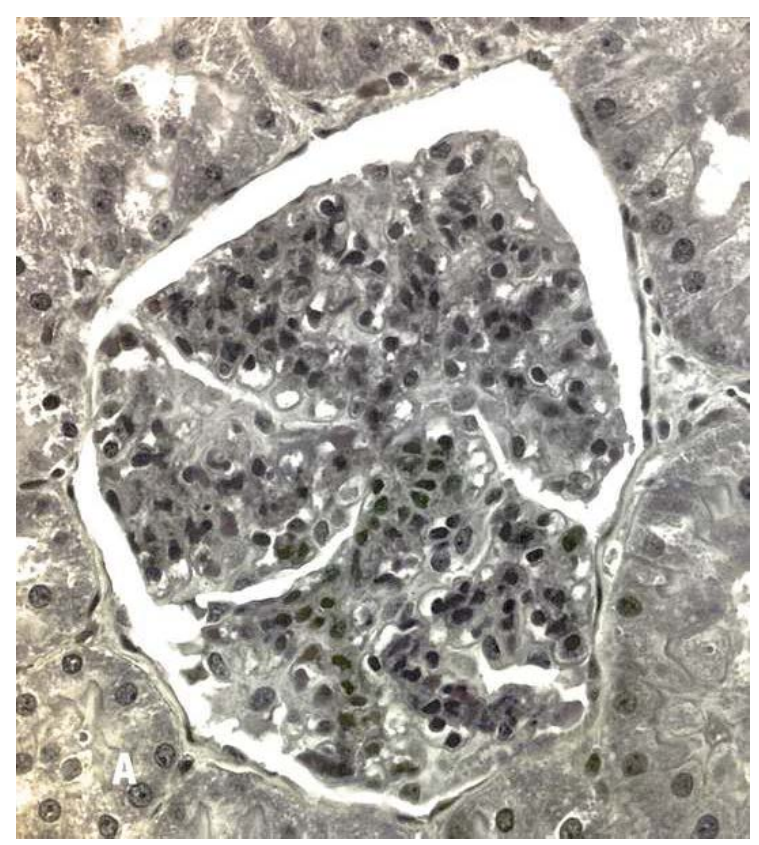

Figure 1A: Pig glomerulus in segmental arterial mediolysis. The glomerulus exhibits the in tandem arrangement of the hyperplastic mesangial cells forming the glomerular stalk. The cytoplasm of these cells and their matrix material has effaced many peripheral capillary loops. Movat $\mathrm{x} 400$. 


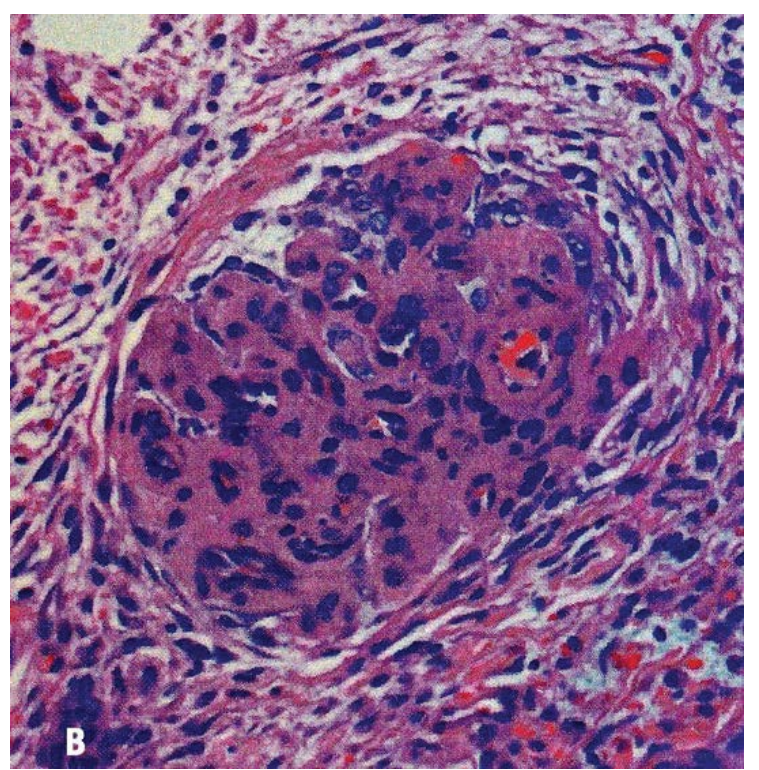

Figure 1B: Greyhound glomerulus in segmental arterial mediolysis. The peripheral capillary loops are severely narrowed or totally obliterated by putatively contracting hyperplastic mesangial cells Hematoxylin \& Eosin $x 400$.

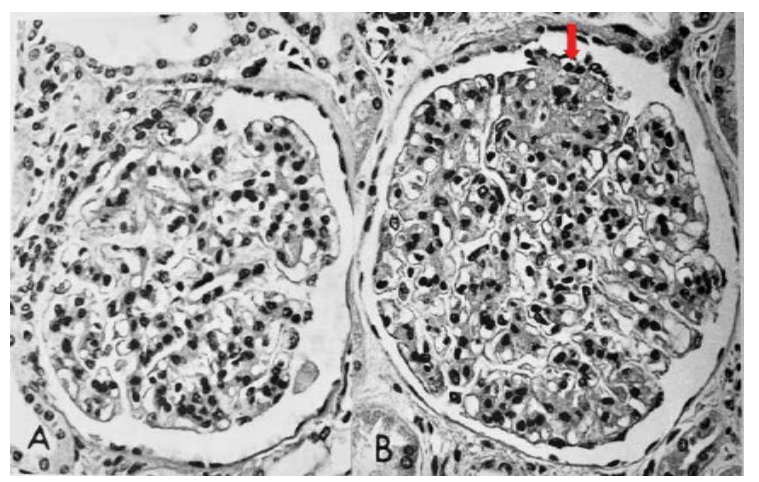

Figure 2: Human glomeruli in segmental arterial mediolysis. Glomerulus in A shows tandem-like arrangement of mesangial cells indicative of hyperplasia. Glomerulus in $\mathrm{B}$, in addition to an increased number of mesangial cells, exhibits a segmental aggregate of mesangial matrix (arrow) obscuring the adjacent glomerular loops. Hematoxylin \& Eosin. Figure A x350, Figure B x300. Adapted with permission from Laboratory Investigation, 35(1), copyright 1976, Nature Publishing Group.

\section{Pathogenesis of SAM}

The segmental distribution of SAM's arterial lesions and its morphologic characteristics in the injurious phase (Figures 3 and 4) favored a vasospastic etiology and the site of the initial lesion in SAM at the outer arterial wall suggested that the responsible vasopressor agent was norepinephrine since it is at this precise site that this agent is produced in varicosities and released from the efferent sympathetic nerve fibers innervating the muscular arteries targeted in SAM [2-6]. This hypothesis was strengthened by the identification of recently administered sympathomimetic agonists in the histories of patients with SAM [6] and the rapid development of SAM in greyhounds following the administration of ractopamine, a Beta- 2 adrenergic agonist used to enhance athletic capabilities and as a repartitioning agent in animal husbandry [8]. This agent also can release norepinephrine from the peripheral nervous system. These findings led to the hypothesis that SAM represented a disease of the peripheral sympathetic nervous system principally induced by the administration of sympathomimetic agonists [10]. Norepinephrine as neurotransmitter in this system couples with the alpha-1 adrenoceptor aggregated on the cell membrane of the smooth muscle cells to induce a conformational change that enabled this receptor to activate a heterotrimeric G-protein. This

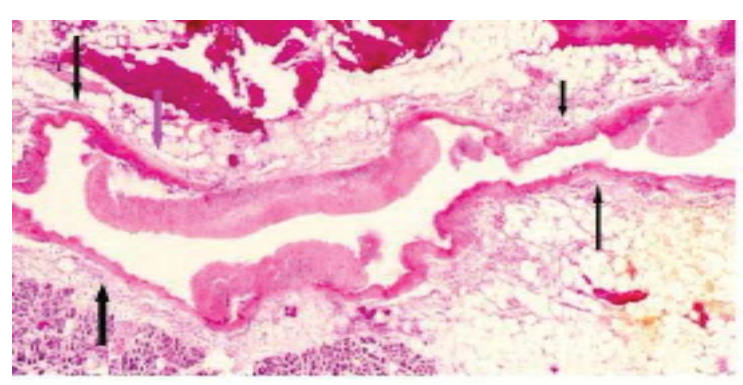

Figure 3: Segmental arterial mediolysis injurious phase. Black arrows point to the segmental distribution of arterial gaps in a longitudinal section of a pancreatic artery. Magenta arrow points to a beginning dissection that will ultimately detach the muscular island to enlarge the gap or form a dissecting hematoma. Hematoxylin \& Eosin x10. Adapted with permission from Laboratory Investigation, 35(1), copyright 1976, Nature Publishing Group.

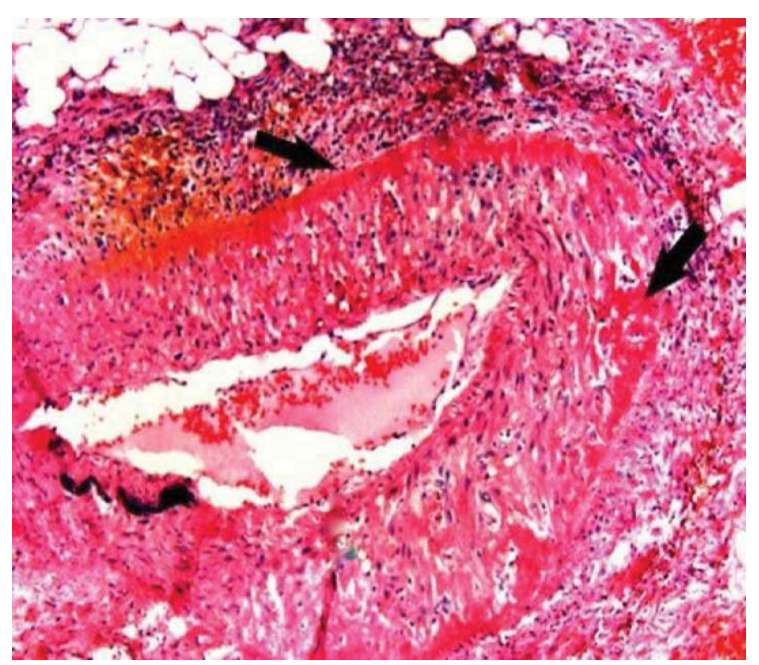

Figure 4: Segmental arterial mediolysis injurious phase. Vasospastic appearance of artery. Black arrows point to thick band of fibrin circumscribing the adventitial medial junction and extending into media to obscure zones of mediolysis. Mononuclear cells, mostly fibroblasts, are evident in the upper periadventitial tissue. Hematoxylin \& Eosin x50. Adapted from the WJCD, 2013(1), published on line. 
initiates a cascade of biochemical events that provokes vasoconstriction and opens calcium channels necessary for this response. These two effects are exaggerated in SAM creating an overzealous vasoconstriction that shears the outer media from the overlying adventitia (Figures 5 and 6) and a cytoplasmic overload of calcium ions that causes mitochondrial dysfunction and injury [11]. The latter is linked to cytochrome $\mathrm{C}$ release that initiates apoptosis (Figure 7) and the increased production of water by reactive oxygen species (ROS) that distends the mitochondria with water causing their rupture with flooding and swelling of the smooth muscle cytoplasm ending in cell membrane rupture (Figures 8-12). These changes create mediolysisthe histologic hallmark lesion of SAM. Trans mural mediolysis with loss of the intima and internal elastica creates arterial gaps (Figures 1 and 13) that dilate to form gap-aneurysms (Figure 14). It is suspected that the stimulus induced by the agonists are intensified

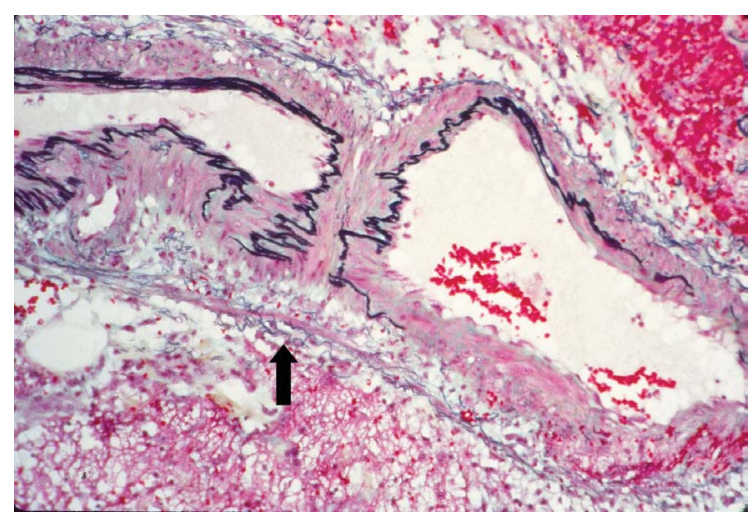

Figure 5: Segmental arterial mediolysis injurious phase. Arrow indicated segment of artery shows a tear separating the outer media from the adventitia. The tear space contains a few intact muscle cells but does not have the foamy appearance of mediolysis. Movat stain $\times 100$. Adapted with permission Cardiovascular Pathology, 18(6), Copyright 2009, Elsevier Inc.

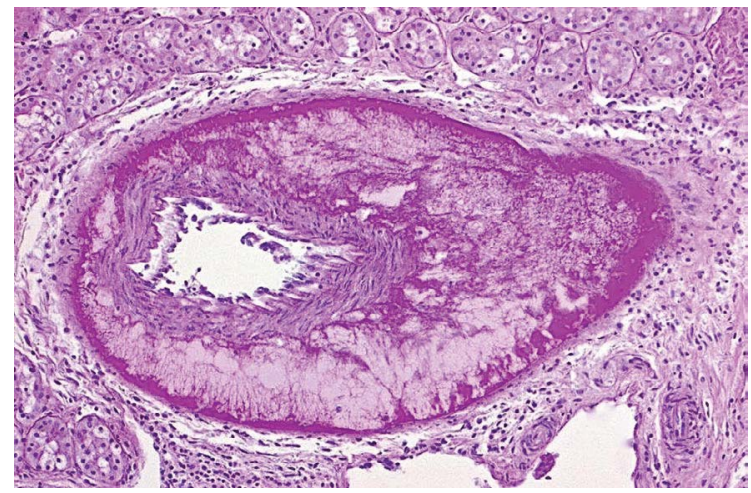

Figure 6: Segmental arterial mediolysis injurious phase. An almost circumferential tear separates the outer media from the fibrin- lined adventitia. The tear space contains edema fluid and fibrin strands. PAS stain $\times 100$.

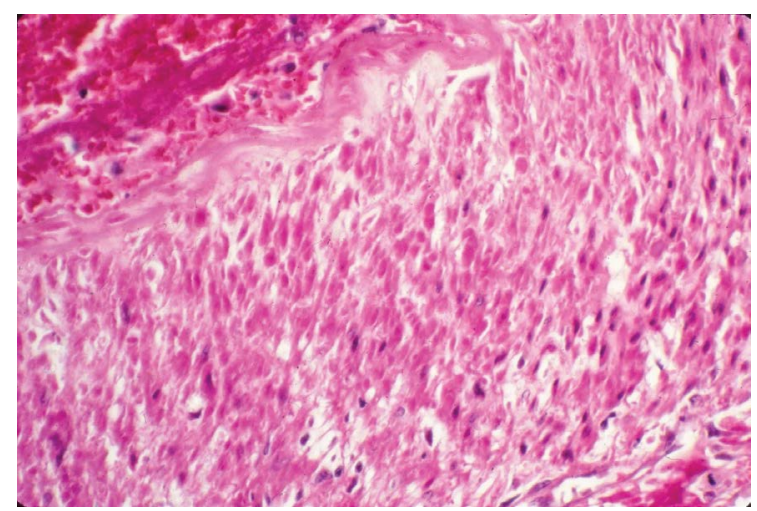

Figure 7: Segmental arterial mediolysis injurious phase: Trans mural apoptosis of medial smooth muscle. Hematoxylin \& Eosin $\mathrm{x} 100$.

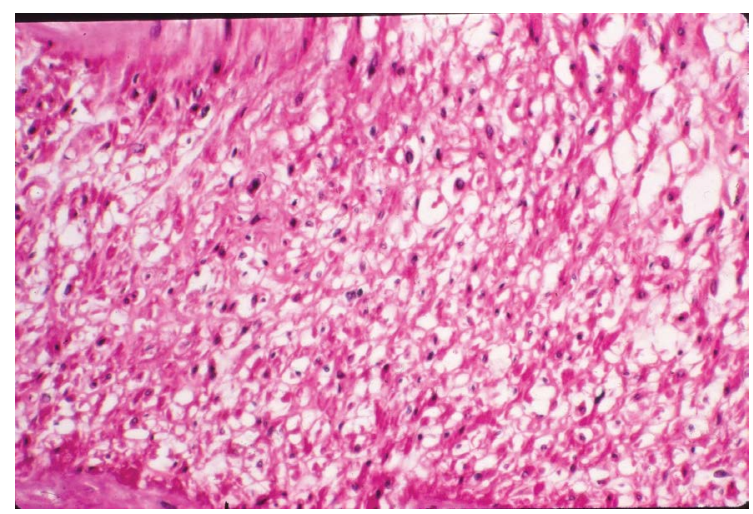

Figure 8: Segmental arterial mediolysis injurious phase. Arterial medial muscle exhibiting the cytoplasmic vacuolar alterations of mediolysis and apoptosis (Figure 9). These changes occurred in the same artery shown in Figure 7. Hematoxylin \& Eosin x200.

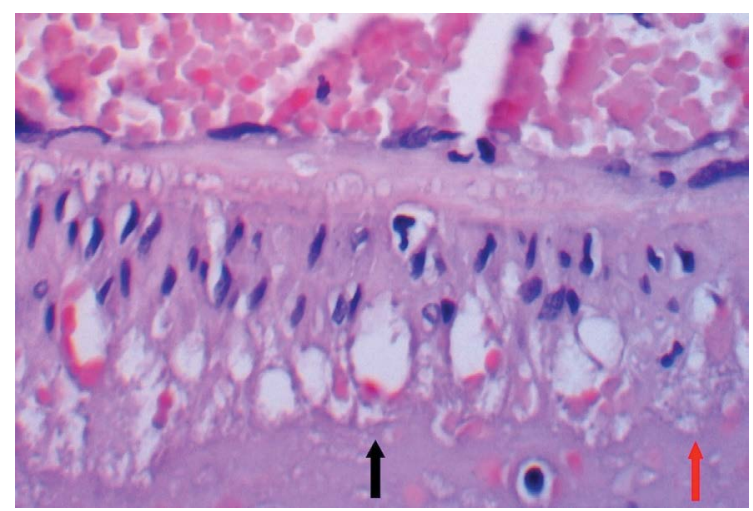

Figure 9: Segmental arterial mediolysis injurious phase. Early phase of mediolysis. Soap-bubble appearance of muscle cells in the outer media created by large cytoplasmic (black arrow) and smaller organelle putatively mitochondrial (red arrow) vacuoles containing clear non-staining contents (water). Hematoxylin \& Eosin $\times 400$. Adapted with permission from Veterinary Pathology, 52(6), copyright 2015, Sage Publications Inc.

in zones of increased density of alpha-1 adrenoceptor distributed on the smooth muscle cell membranes [10]. This receptor is in a dynamic state and its density is augmented by factors such as age- increased in the 


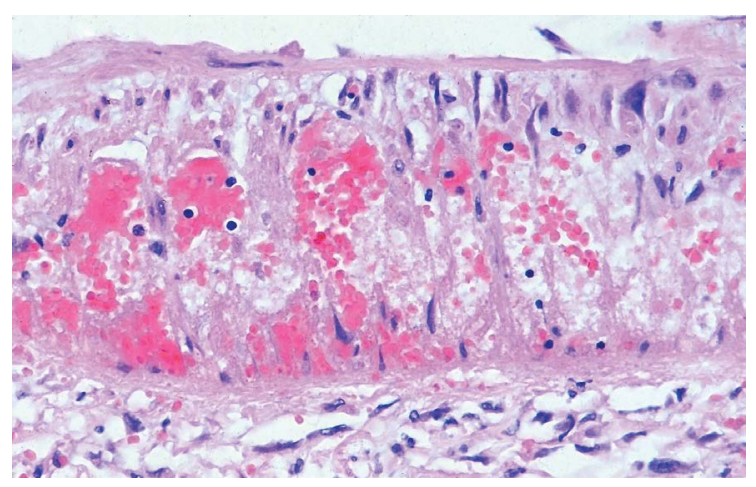

Figure 10: Segmental arterial mediolysis injurious phase. Arterial media exhibiting confluent zones of mediolysis involving outer and mid and focally the inner media. Areas of mediolysis have a foamy appearance caused by organelle and membranous debris and exhibit clusters of red cells and sparse inflammation. Scattered intact muscle cells are distributed in areas of mediolysis. A linear deposit of fibrin focally lines the adventitia. Hematoxylin \& Eosin x200. Adapted from the WJCD, 2013(1), On-line publication.

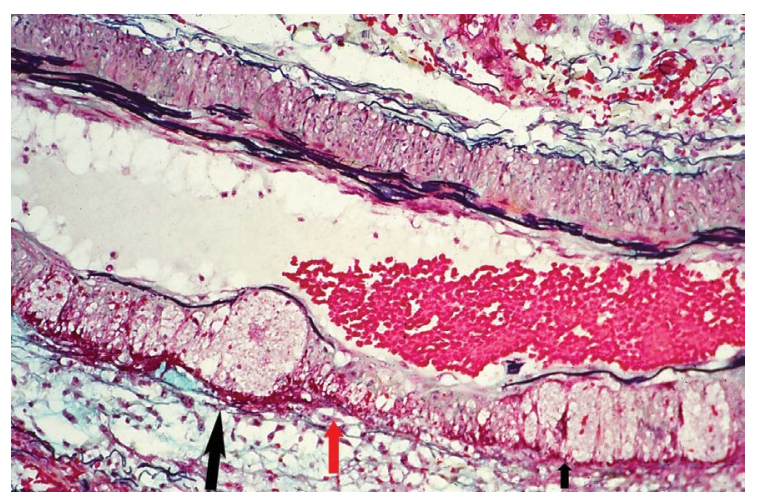

Figure 11: Segmental arterial mediolysis injurious phase. Large black arrow points to a distended area of total mediolysis putatively caused by water that has caused an outward bowing of both the preserved internal elastic (inner black membrane) and the fibrin lined (red stained) adventitia. Smaller black arrow indicates another area of somewhat edematous trans mural confluent mediolysis. Note the foamy appearance of the mediolytic areas. Red arrow points to an arterial area showing loss of the internal elastica and partial loss of the edematous media. Movat stain $\times 100$. Adapted with permission from the American Journal of Surgical Pathology, 13(7), copyright 1989, Lippincott, Williams and Wilkens.

elderly, sex-elevated in premenopausal females and possibly also by previous exposure to sympathomimetic agents-all important clinical considerations in patients developing SAM [12,13]. This dynamism explains why abdominal, basilar and pericardial arteries are targeted in different age groups. The plasticity of this receptor permits the generation of discrete clusters of hyper density explaining SAM's capricious arterial involvement within each of these arterial beds. Prolonged and/or excessive release of norepinephrine may also contribute to the extensiveness of mediolysis since only the outer medial smooth muscle cells receive

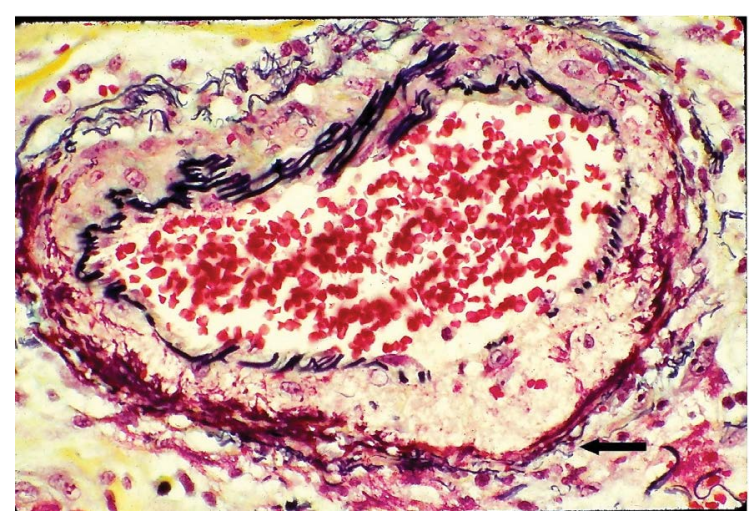

Figure 12: Segmental arterial mediolysis injurious phase. Arrow points to a focus of confluent trans medial mediolysis. It foamy watery content has caused an outward bowing of the fibrin suffused adventitia. The internal elastica is fragmented with a loss of intima. These changes will eventually allow the medial contents to be evacuated into the bloodstream creating the arterial gaps visualized in Figures 3 and 13. Movat stain $\times 50$. Adapted with permission from the American Journal of Surgical Pathology, 13(7), copyright 1989, Lippincott, Williams and Wilkens.

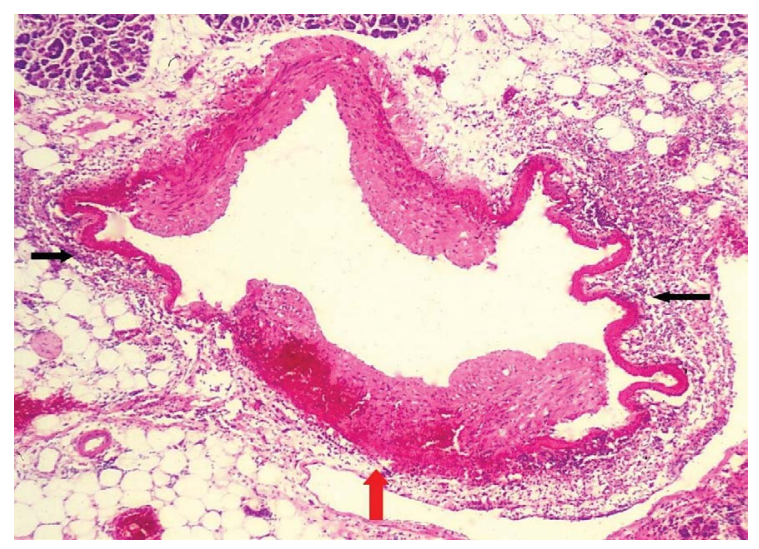

Figure 13: Segmental arterial mediolysis injurious phase. Artery shows 2 gaps (black arrows) bordered by a thick linear band of fibrin. Lower medial island (red arrow) is being detached from the adventitia by blood tracking between the outer media and adventitia and bleeding from distended capillaries in the outer media. The loss of this medial island will significantly enlarge this gap. Hematoxylin \& Eosin $\times 100$. Adapted with permission from the International Journal of Surgical Pathology, 15(2) copyright 2007, Sage Publications, Inc.

sympathetic innervation while the inner medial muscle cells are exposed to norepinephrine by diffusion and so may be targeted or spared depending upon the quantity of released norepinephrine.

The hyper activated $\mathrm{Cq}$ heterotrimeric $\mathrm{G}$ protein and the surfeit of calcium ions is also putatively responsible for the robust rapid proliferation of granulation tissue developing as a response to the mediolysis and adventitial medial tears (Figures 15 and 16). The extracellular matrix produced by these tissue repairs non-extensive arterial lesions (Figure 17) and stabilizes 


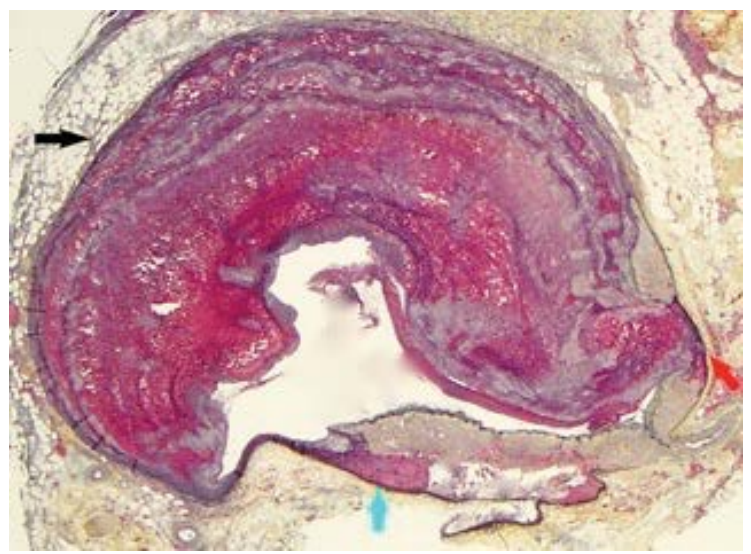

Figure 14: Segmental arterial mediolysis injurious phase. Multiplicity of lesions-black arrow points to large gap-aneurysm partially filled by a recent thrombus, magenta arrow points to a dissection and red arrow points to a small gap. Movat stain x50. Adapted with permission from Modern Pathology, 18(3), copyright 1995, Nature Publishing group.

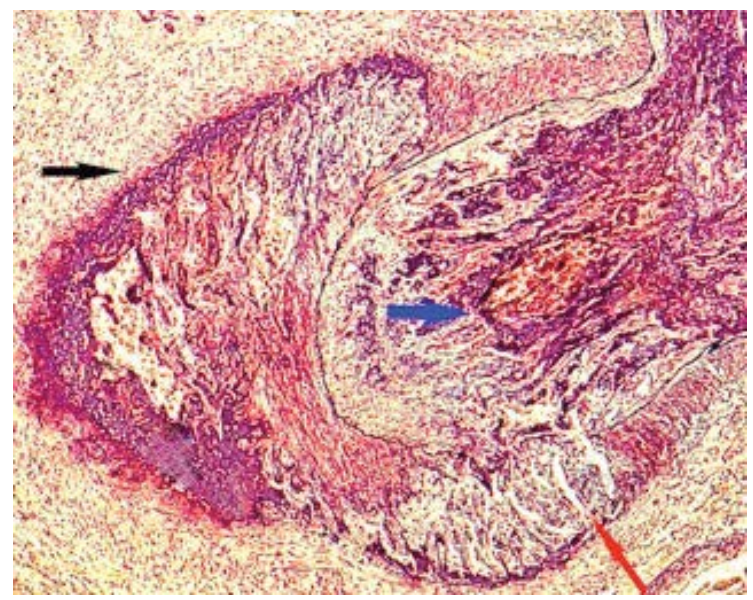

Figure 15: Segmental arterial mediolysis reparative phase (early). Red arrow points to granulation tissue filling and expanding a focus of trans mural mediolysis and penetrating the fragmented internal elastica to form an intimal plaque that significantly narrows the arterial lumen blue arrow already partially occluded by a recent thrombus. Black arrow points to another focus of watery confluent trans mural mediolysis that pushes the thick linear fibrin lined adventitia outwardly. The top of this area shows another focus of newly forming granulation tissue. Movat stain $\times 100$. Adapted from the Journal of Cardiovascular Diseases \& Diagnosis 2015, 3:2. On-line publication.

small gap- aneurysms but more importantly produce sequelae. These include arterial stenosis (Figures 15 and 18), persistent large arterial aneurysms (Figure 19), dissecting hematomas (Figure 20), and fibromuscular dysplasia (Figures 21 and 22).

\section{The Mesangium}

The mesangial cell is a myofibroblast with phagocytic capabilities originating from hematopoietic stem cells [14]. These morphologic characteristics enable the mesangial cells and their matrix to provide central

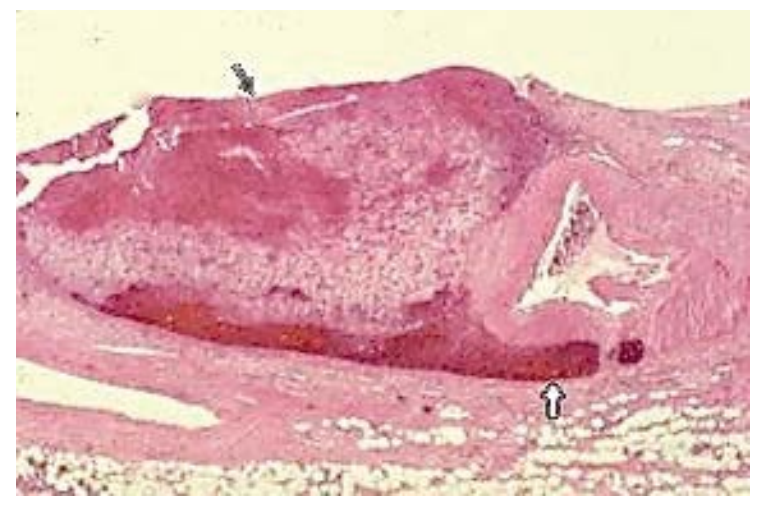

Figure 16: Segmental arterial mediolysis reparative phase. Black arrow points to a florid proliferation of young granulation tissue in the space created by the tearing separation of the outer media from the adventitia. Capillary bleeding has initiate a dissecting hematoma (white arrow) tracking between the outer media and the adventitia. Hematoxylin \& Eosin x15. Adapted with permission from the International Journal of Surgical Pathology, 15(2), copyright 2007, Sage Publications, Inc.

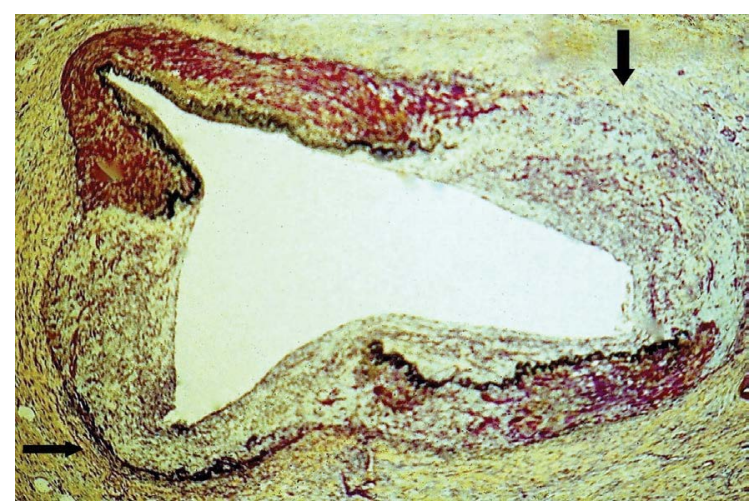

Figure 17: Segmental arterial 'mediolysis reparative phase. Arrows points to two large foci of trans mural mediolytic repair by maturing granulation tissue that has focally extended over the intima to form a thin intimal plaque. Arteries with similar reparative alterations may appear normal in radiologic studies. Movat stain x100.

axial support and stability for the capillary loops of the glomerulus and acting as macrophages to perform a house keeping function by removing trapped residues and aggregated proteins including immune complexes from the glomerular basement membrane. Finally and most importantly for this discussion is the ability of mesangial cells to regulate blood flow of the glomerular capillaries by their contractile activity. The mesangial cell is the glomerular homologue of vascular smooth muscle [15]. It expresses alpha- 1 adrenoceptors and the biochemical events initiating its contraction are similar to those occurring in smooth muscle $[15,16]$. Norepinephrine causes a decrease in the cellular surface area of the glomerulus consistent with mesangial contraction and it also directly modulates extracellular matrix protein synthesis $[15,16]$. The mesangial cells, like the renal arteries are innervated by the peripheral sympathetic nervous system. 


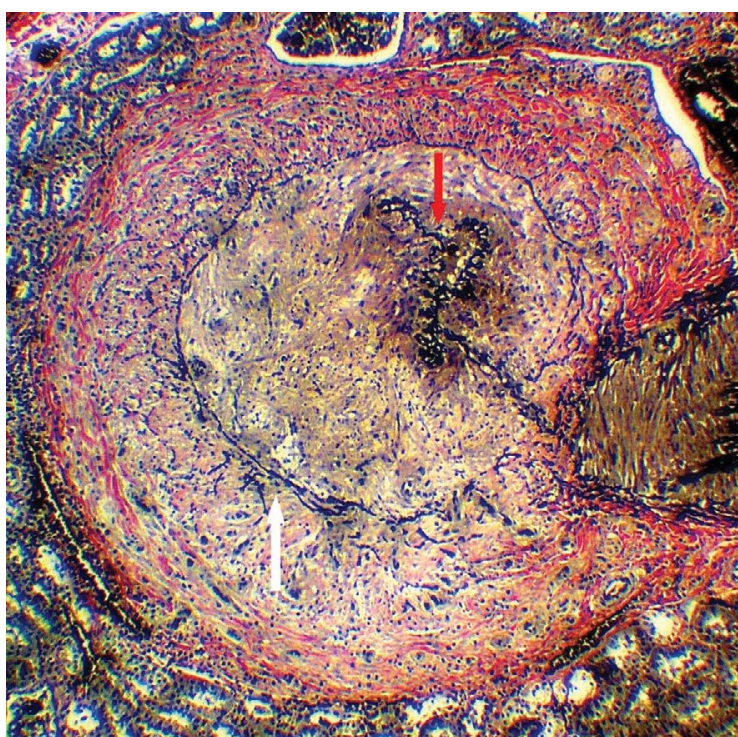

Figure 18: Segmental arterial mediolysis reparative phase. Renal artery stenosis caused by luminal compression by granulation tissue circumferentially developing in the adventitia and between the external elastica (white arrow) and lumen outlined by black staining internal elastica (red arrow). Verhoef van Gieson x100. Adapt with permission from Veterinary Pathology, 52(6), copyright 2015, Sage Publications Inc.

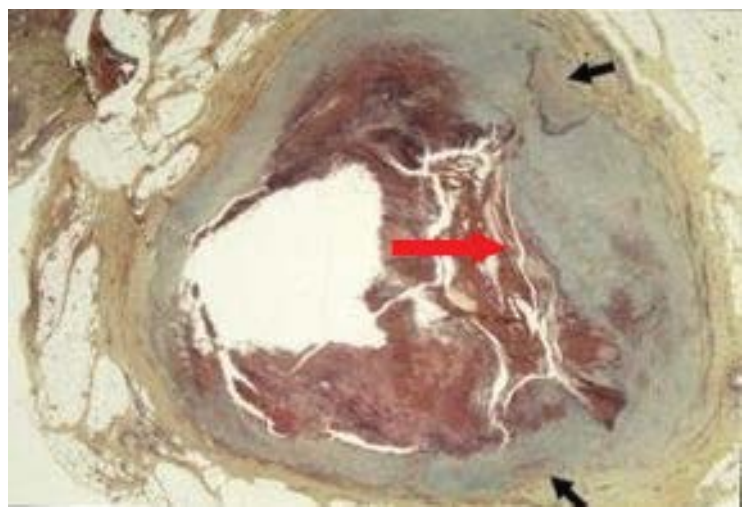

Figure 19: Segmental arterial mediolysis reparative phase. Red arrow points to a thick layer of granulation tissue filling a large gap and extending over the intima of the surviving small medial islands (black arrows) to encircle the adjacent gap-aneurysm. Movat stain $\times 25$. Adapted with permission from Cardiovascular Pathology, 18(6), copyright 2009, Elsevier Inc.

We suspect that the same agonists that cause SAM also impacts the sympathetic nervous system that innervates the bone marrow to stimulate the mesangial stem or progenitor cells to form mesangial cells and to rapidly release them to colonize the glomeruli. Norepinephrine released from the sympathetic nervous system regulates the migration of hematopoietic stem \& progenitor cells from the bone marrow to their extra medullary niches $[17,18]$. The stimulus provided by these agonists is probably intense but short lived since only a few glomeruli are populated similarly to

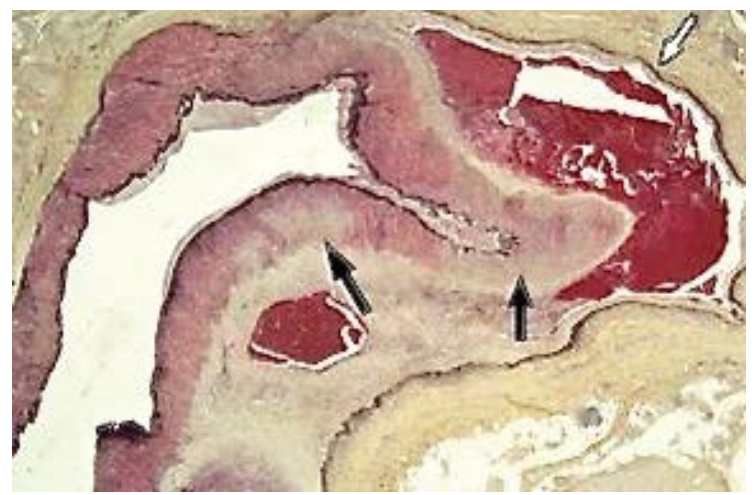

Figure 20: Segmental arterial mediolysis reparative phase. Dissecting hematoma (white arrow) and hematoma developed in the mature granulation tissue filling and expanding the tear space between the outer media and the adventitia. Black arrows point to foci of mediolysis repaired by mature granulation tissue. Movat stain $\times 15$. Adapted with permission from the International Journal of Surgical Pathology, 15(2), copyright 200, Sage Publications, Inc.

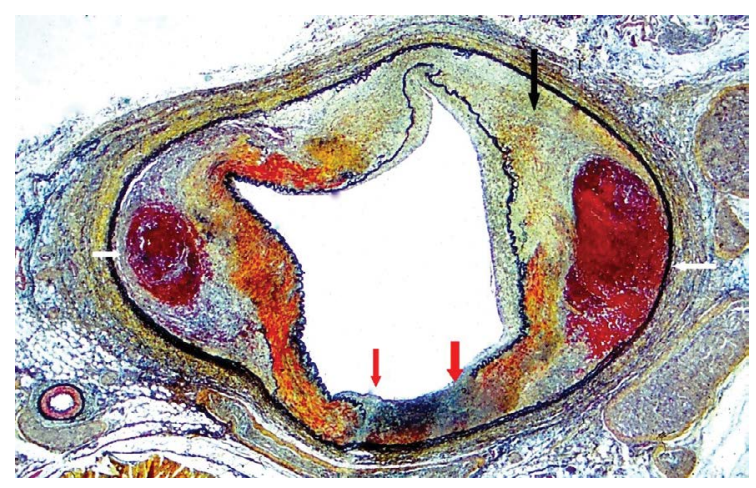

Figure 21: Segmental arterial mediolysis reparative phase evolving into fibromuscular dysplasia. Two hematomas (white arrows) flanking the arterial lumen are evident. These were created from bleeding capillaries in the granulation tissue (black arrow) that developed in the adventitial-medial tear space and expanded into zones of mediolysis. The internal elastica is focally lost over a gap repaired by granulations tissue (red arrows) and this is bedecked by a thin intimal plaque that focally extends over the adjacent intima. The surviving media stained orange -brown shows areas of fibrosis (yellow stain). Movat stain x25. Adapted with permission from Cardiovascular Pathology, 18(6), copyright 2009, Elsevier Inc.

the few arteries targeted in SAM. The stimuli to the peripheral sympathetic system in the kidneys that provokes SAM also putatively releases norepinephrine that forms conformational changes with the alpha- 1 adrenoceptors on the cell membrane of the mesangial cells to cause these cells to contract. In SAM, the contraction is exaggerated causing mediolysis and the shearing separation of the outer media from the adventitia while in the glomerulus the mesangial cell contraction narrows and obliterates the peripheral glomerular capillaries. The exaggerated response injuries occurring in the arteries was not evident in the 


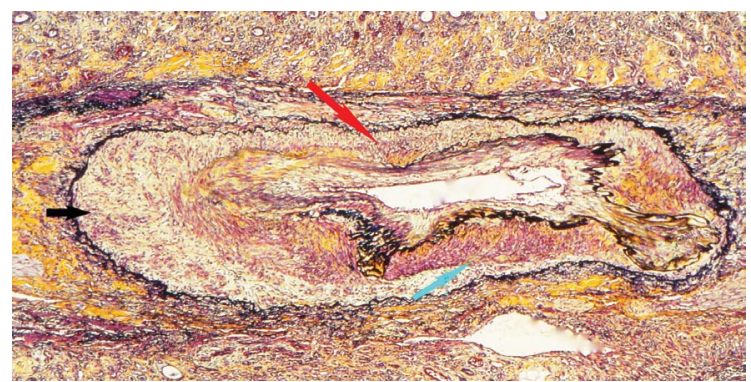

Figure 22: Segmental arterial mediolysis reparative phase evolved to fibromuscular dysplasia. Black arrow points to granulation tissue filling and expanding tear space between outer media and adventitia (outlined by black staining external elastica) and expanding into and replacing areas of medial loss through mediolysis (red arrow). Cyan arrow points to residual intact medial muscle (brown stain) showing patchy fibrosis (yellow stain). The internal elastica (inner black staining membrane) is patchily absent in areas of trans medial muscle loss. A circumferential plaque of granulation tissue that narrows the arterial lumen bedecks the intima. Movat stain $\times 25$. Adapted with permission from Cardiovascular Pathology, 18(6), copyright 2009, Elsevier Inc

mesangial cell i.e. mediolysis or apoptosis of mesangial cells was absent in the swine, dog or human glomeruli examined in this study. Like the dysfunctional sparse arterial involvement in SAM the norepinephrine induced contraction of the mesangial cells found in humans and dogs was also putatively dysfunctional since too few glomeruli had the hyperplasia necessary to significantly decrease the total glomerular blood flow and filtrating surface areas of its capillary component. Indeed in many human cases it was not detected.

The mesangial cell hyperplasia was more striking in the pig. Its and SAM's stimuli were not certain. Possible iatrogenic agonists responsible for SAM were not identified in these pigs. The usage of the repartitioning agent ractopamine is illegal in Denmark. The SAM renal pig lesions were injurious phase with focal concomitant repair consistent with the time interval required for the pig's round-up and final journey to and stay in the abattoir before slaughter. In humans, injurious SAM lesions can be identified as early as 3 hours following the administration of vasopressor agonists [19]. These observations suggested that SAM and the mesangial cell hyperplasia could represent collateral lesions in a fightflight response [9]. Could this adrenal medulla directed defensive generalized response, caused by circulating norepinephrine stimulate the peripheral sympathetic nervous system to release norepinephrine in the hilar and large intra-renal arteries in order to constrict the renal circulation and mobilize mesangial cells from the bone marrow to the glomeruli and cause their contraction decreasing intraglomerular blood volume and urine production? Such physiological responses could rapidly divert blood away from the kidneys to the skeletal muscle and because of their focal nature also protect the kidney from developing extensive ischemic injury. However, because the release of norepinephrine in these pigs putatively was supra physiological it caused SAM. SAM and mesangial cell hyperplasia to our knowledge has not been reported in the "flight-fight response" but pathologic studies of this response are scanty.

The flight and fight response as a potential cause of SAM suggested that SAM may also develop from other endogenous pathologic states causing or associated with supra-physiologic elevations of norepinephrine. Pheochromocytomas are a prime example of this phenomenon. These tumors manufacture excessive catecholamine's (epinephrine and norepinephrine) that are released in a continuous or episodic manner. Eightyseven cases of pheochromocytomas have been reported to have coexisting renal artery aberrations including stenosis, arterial fibromuscular dysplasia, aneurysms and dissection with occlusion [19]. The co-existence of these two rare entities is more than fortuitous. Renal artery aberrations were identified in $3.7 \%$ of 269 cases of pheochromocytomas in a study reported by Gill and coworkers and in $7 \%$ of pheochromocytomas / parganglioma in an article by Sarathi and coworkers $[20,21]$. The most common cause for the renal artery lesions was the extrinsic compression of the renal artery by the tumor but this was only present in $36 \%$ of cases [20]. Other attributable causes were the simultaneous but independent occurrence of stenotic renal artery lesions such as atheromatous plaques, catecholamine-induced spastic vasoconstriction and arterial fibromuscular dysplasia. The latter two proposed etiologies are consistent with the development and repair of SAM. SAM was not reported in these studies because ruptured gap-aneurysms with massive hemorrhages, the principle clinical expression of SAM chiefly occurs in the elderly while pheochromocytomas are tumors mainly developing in young and middle aged adults [20]. Moreover, injurious phase SAM develops very rapidly and is short-lived and, aside for the development of gap-aneurysms, is usually initially asymptomatic. These considerations militate against the simultaneous discovery of clinically expressed SAM, in its injurious phase, with a diagnosed pheochromocytoma. The episodic or continuous circulating supra-physiologic release of catecholamines by this tumor may "sensitize" the arterial smooth muscle by promoting movement of the alpha-1 adrenoceptors creating the hyper-dense areas that are believed instrumental for the development of SAM 
[10]. The close proximity of the pheochromocytomas with its released circulating catecholamines to the celiac plexus could also expedite this sensitization.

\section{Conclusion}

The nexus of SAM is norepinephrine. Morphologic and clinical features described above link this hormone to the pathogenesis of this vascular disorder. Iatrogenic administered sympathomimetic drugs, Beta-2 agonists including meat laced with the repartitioning agent ractopamine, all having the ability to liberate norepinephrine from ganglia on the efferent branches of the sympathetic nervous system innervating the targeted arteries, have been implicated in its genesis $[6,8]$. These stimuli, however, were not always evident in many reported cases of SAM and their absence cast doubt on this hypothesis. The realization that SAM may also occur in a fight and flight response and as a collateral lesion in pheochromocytomas suggest that circulating supra physiologic levels of norepinephrine produced by the adrenal medulla also may act as a neurotransmitter in the peripheral sympathetic nervous system to cause SAM and its sequelae. This alternate, principally stress induced pathogenic pathway, would explain the pathogenesis of SAM and its sequelae, particularily fibromuscular dysplasia, in histories devoid of any iatrogenic sympathomimetic agonist stimuli. The various stimuli causing SAM may also provoke focal mesangial cell hyperplasia and contraction as incidental collateral lesions. Could these changes, like the hypertrophy and hyperplasia of the mesangial cells caused by angiotensin II [22] also represent the precursors of focal segmental glomerulosclerosis especially since matrix deposition also is stimulated by the peripheral sympathetic nervous system [23]? Indeed, the microphotograph of the glomeruli with hyperplasia in reference 1 (Figure 2) show focal sclerosis. The identification of co-existing arterial reparative changes of SAM in cases of glomerulosclerosis would further strengthen this hypothesis and add disorders of the peripheral sympathetic nervous system to the multiple etiologies of this renal disease.

\section{Acknowledgements}

The authors would like to thank the veterinary pathologist Micheal Yaeger for making available slides of greyhounds fed ractopamine, Beth Caldwell of the Legacy LSO \& CME services for aid with the references and Jason Slavin for his assistance in formatting the digital images. No funding agent played a role in the preparation or writing of this manuscript.

\section{Executive summary}

Background: Segmental arterial mediolysis (SAM) a rare arteriopathy causing massive bleeding or ischemic symptoms, is suspected of representing a vascular disease of the peripheral sympathetic nervous system. It is initiated by the supra physiological release of norepinephrine from the efferent branches of the peripheral sympathetic nerves that innervate the large and medial sized muscular arteries targeted in SAM. Recognized stimuli for this response are iatrogenic sympathomimetic agonists and some B-2 agonists. However, these stimuli were not always apparent in published cases of SAM casting doubt on this hypothesis.

Methods and findings: SAM, reported in kidneys of slaughtered pigs, was believed to represent a dysfunctional development in a fight and flight response. Additionally some stenotic renal arteries described in cases of pheochromocytomas reportedly were caused by arterial spasm and fibromuscular dysplasia changes found in the evolution of SAM. Varying degrees of mesangial cell hyperplasia accompanied SAM in swine, dog and scattered human cases of SAM. Segmental sclerosis of glomerular loops accompanied the mesangial cell hyperplasia in a few cases of SAM. The hyperplasia was not extensive so that its role in regulating blood flow and urine volume through glomerular capillaries was probably inconsequential. It represented a collateral lesion created by SAM's norepinephrine driven pathogenesis.

Conclusion: These findings provide indirect evidence that SAM additionally may be initiated by conditions causing the adrenal medulla to release supra physiologic levels of circulating norepinephrine. Supra physiologic release of norepinephrine from the peripheral sympathetic nerves also can cause mesangial hyperplasia that can be accompanied with segmental glomerular loop sclerosis-making it another precursor lesion of focal segmental glomerulosclerosis. 


\section{References}

1. Slavin RE, Gonzalez-Vitale. Segmental mediolytic arteritis a clinical pathologic study. Lab. Invest. 35(1): 23-29 (1976).

2. Slavin RE, Kazunori S, Bhagavan B, Maas AE. Segmental arterial mediolysis: a precursor to fibromuscular dysplasia? Mod. Pathol. 8(3): 287-294 (1995).

3. Slavin RE, Cafferty L, Cartwright J. Segmental mediolytic arteritis: A clinicopathologic and ultrastructural study of 2 cases. Am. J. Surg. Pathol. 13(7): 558-568 (1995).

4. Slavin RE, Inada K. Segmental arterial mediolysis with accompanying venous angiopathy: a clinical pathologic review, report of 3 new cases, and comments on the role of endothelin-1 in its pathogenesis. Int. J. Pathol. 15(2): 121-134 (2007).

5. Slavin RE. Segmental arterial mediolysis: course, sequelae, prognosis, and pathologic-radiologic correlation. Cardiovasc. Pathol. 18(6): 352-360 (2009).

6. Slavin RE. Segmental arterial mediolysis: A clinical-pathologic review, its role in fibromuscular dysplasia and description and differential diagnosis of the masquerader-muscular artery cystic necrosis. WJCD. 3: 64-81 (2013).

7. Slavin RE, Gonzalez JC, Machin JM, Robles A, Regojo RM, Diez ML. Segmental arterial mediolysis, reparative phase: An analysis and case report showing conversion to fibromuscular dysplasia with renal infarction. WJCD. 4: 50-60 (2014).

8. Slavin RE, Yaeger MJ. Segmental arterial mediolysis- an iatrogenic vascular disorder induced by ractopamine. Cardiovasc. Pathol. 21(4): 334-338 (2012).

9. Leifsson PS, Slavin RE. Segmental arterial mediolysis in pigs presenting with renal infarcts. Vet. Pathol. 52(6): 1157-1162 (2015).

10. Slavin RE. Segmental arterial mediolysis: A review of a proposed vascular disease of the peripheral sympathetic nervous system-A density disorder of the alpha-1 adrenergic receptor? J. Cardiovasc. Dis. Diagn. 3(2): 2-7 (2015).

11. Brookes PS, Yoon Y, Robotham JL, Anders MW, Sheu SS. Calcium, ATP and ROS: a mitochondrial love-hate triangle. Am. J. Physiol. 287(4): C817-833 (2004).
12. Rudner XL, Berkowitz DE, Booth JV, et al. (1999) Subtype specific regulation of human vascular alpha-1 adrenergic receptors by vessel bed and age. Circulation. 100: 2336-2343.

13. Anesiri C, Borda E. Hormonal influence on expression and functionality of alpha-adrenoceptor in rat submandibular gland. Auton. Neurosci. 103: 13-18 (2003).

14. Masuya M, Drake CJ, Fleming P, et al. Hematopoietic origin of glomerular mesangial cells. Blood. 101(6): 2215-2218 (2003).

15. Pawluczyk IZA, Patel SR, Harris KPG. The role of the a-1 adrenoceptor in modulating human mesangial cell matrix production. Nephrol. Dial. Transplant. 21(9): 2417-2424 (2006).

16. Jackson EK. Autonomic control of the kidney: Primer on the autonomic nervous system, Ed Robertson D, Biaggioni I, Burnstock G, Low PA, (2nd Ed). Elsevier. Academic. Press. Amsterdam (2004).

17. Kon V, Karnovsky MJ. Morphologic demonstration of adrenergic influences on the glomerulus. Am. J. Pathol. 134(5): 1039-1046 (1989).

18. Katayama Y, Battista M, Kao WM, et al. Signals from the sympathetic nervous system regulate hematopoietic stem cell egress from bone marrow. Cell. 124(2): 407-421 (2006).

19. Felipe-Silva A, Ferraz de Campos FP, Martines JAS. Fatal hemoperitoneum due to segmental arterial mediolysis. Autops. Case. Rep. 6(3): 7-15 (2016).

20. Gill IS, Meraney AM, Bravo EL, Novick AC. Pheochromocytoma coexisting with renal artery lesions. J. Urol. 164: 296-301 (2000).

21. Sarathi V, Bandgar T, Lila AR, et al. Coexistence of pheochromocytoma/paraganglioma and renal artery stenosis. Indian. J. Endocrinol. Metab. 16(4): 1009-1011 (2012).

22. Orth SR, Weinreich T, Bonisch S, Weih M, Ritz E. Angiotensin 11 induces hypertrophy and hyperplasia in adult human mesangial cells. Exp. Nephrol. 3(1): 23-33 (1995).

23. Micallef L, Vedrenne N, Billet F, Coulomb B, Darby IA, Desmouliere A. The myofibroblast, multiple origins for major roles in normal and pathological tissue repair. Fibrogenesis. Tissue. Repair. 5: 55 (2012). 